\title{
PERIODONTAL INFECTION AND PRETERM BIRTH: HOW STRONG IS THE EVIDENCE?
}

Christine L. Roberts

Research Director

NSW Centre for Perinatal Health Services Research

\section{Charles S. Algert}

Research Associate

NSW Centre for Perinatal Health Services Research

A recent NSW Public Health Bulletin article discussed the possibility that periodontal infection may be a risk factor for preterm birth. ${ }^{1}$ One of the papers cited in support of this association was 'Periodontal infection as a possible risk factor for preterm low birth weight' by Offenbacher et al. ${ }^{2}$ Such an association is potentially of major importance. In Australia, approximately seven per cent of births are preterm each year, and for many of these there is no known cause. ${ }^{3,4}$ Should an infectious cause be found, this would be a tremendously exciting result as, other than smoking, most risk factors for preterm birth are not modifiable. However, we believe the Offenbacher article provides only weak evidence, if any, of a causal association between periodontal disease and preterm birth because of significant methodological problems in the study.

Offenbacher's study results suggest that periodontal disease may be a risk factor for preterm low birthweight (PLBW) infants. But what the study literally shows is a very strong association between PLBW and a variable the authors have created, Extent 3:60. This is a yes/no variable of dental disease, 'yes' meaning that a woman had clinical attachment loss (CAL) of 3+ mm affecting 60 per cent or more of her dental sites. CAL is a measure of the loss of support, both soft tissue (gum) and hard tissue (bone) around a tooth caused by periodontal disease. It is measured in millimetres and is the distance from the junction between the enamel (covering the crown of the tooth) and the cementum (covering the root of the tooth) and the base of the periodontal pocket (the point where the gum meets the tooth root). ${ }^{5}$

The authors seem to suggest that the Extent 3:60 variable was selected in order to have a tighter confidence interval around their result. The cases in the study had an adjusted odds ratio (aOR) of 7.5 for having Extent 3:60. However, the authors state that 'mean CAL measures are generally an insensitive measure of disease'. An expert opinion on the study factors (periodontal disease, how it is assessed, other causes and its relationship with infection and antibiotics) would be of considerable interest.

The other evidence from the study for an association between periodontal disease and PLBW is not nearly as dramatic. The differences in extent and severity of clinical attachment loss between cases and controls were not great when reported as continuous variables and were marginally statistically significant.

Other methodological questions hover over this study. Unusually, case status is defined as preterm birth in the current or a previous pregnancy plus birthweight $<2500$ g. In NSW, only 60 per cent of preterm infants are $<2500$ $\mathrm{g}$, and it is unclear why the authors have chosen to select on size as well as maturity. Although called a case control study, it appears to be a convenience study on a group of volunteers from the University of North Carolina (UNC) Prenatal Care Clinic. The women in the study differ from the UNC study base, with black women markedly overrepresented. Maternal race was the only factor described for the entire UNC population. The authors also state that 'The mean age for cases was ... not significantly different from controls.' Yet a comparison of the mean ages and standard deviations shows that the controls were significantly younger than the cases $(p<0.01)$. All of this raises questions as to how the controls were selected and how representative they are of the general population.

There were only 31 controls for the 93 cases in this study, an unusually low ratio. It would appear that 16 of the controls were recruited at an antenatal clinic, and 12 of these had their periodontal assessment then. The examiners could hardly have been blinded to the likelihood that these pregnant mothers would be controls. Another difficulty is with the reported protective effect of cystitis and bacterial vaginosis, both risk factors for preterm birth. ${ }^{6,7}$ The authors speculate that the strongly protective effect of a history of cystitis ( $\mathrm{aOR}=0.15,95$ per cent CI 0.02-0.92) or the presence of bacterial vaginosis in the current pregnancy $(\mathrm{aOR}=0.10,95 \mathrm{per}$ cent CI 0.01-1.74) is due to the use of systemic antibiotics for these conditions. However, this appears to contradict the methods that indicate that women treated with antibiotics during the current pregnancy were excluded.

Although it is stated that data were collected, other risk factors for preterm birth (multiple pregnancy, antepartum haemorrhage, pregnancy-induced hypertension etc) that may confound the relationship with Extent 3:60 are not described, nor are they controlled for in the analysis.

In summary, the Offenbacher study provides evidence of a strong association between PLBW and a constructed outcome variable, Extent 3:60, among a group of mothers who may or may not be representative of the general population. It also seems to provide evidence that a history of [treated] cystitis or bacterial vaginosis is protective against PLBW. Clearly these results need to be interpreted with caution. Because preterm birth is an important problem, further research is warranted to see if these results are repeatable by other investigators. 


\section{REFERENCES}

1. Taylor BA. Periodontal diseases and systemic health: Associations, directions, implications. NSW Public Health Bulletin 1998; 10: 14-16.

2. Offenbacher S, Katz V, Fetik G et al. Periodontal infection as a possible risk factor for preterm low birth weight. $J$ Periodontol 1996; 67: 1103-1113.

3. Day P, Sullivan E, Lancaster P. Australia's mothers and babies 1996. AIHW Cat. No. PER 4. Sydney: Australian Institute of Health and Welfare National Perinatal Statistics Unit (Perinatal Series No. 7).
4. Savitz DA, Blackmore CA, Thorp JM. Epidemiologic characteristics of preterm delivery: Etiologic heterogeneity. Am J Obstet Gynecol 1991; 164: 467-71.

5. Beck JD, Loe H. Epidemiological principles in studying periodontal diseases. Periodontology 2000 1993; 2: 34-45).

6. Romero R, Oyarzun E, Mazor M et al. Meta-analysis of the relationship between asymptomatic bacteriuria and preterm delivery/low birth weight. Obstet Gynecol 1989; 73: 57682.

7. Hay PE, Lamont RF, Taylor RD et al. Abnormal bacterial colonisation of the genital tract and subsequent preterm delivery and late miscarriage. BMJ 1994; 308: 295-8.

\section{CUPID AND THE TOOTH FAIRY: THE LINK BETWEEN CARDIOVASCULAR DISEASE AND ORAL HEALTH}

Geoffrey H. Tofler

Professor of Preventive Cardiology, University of Sydney

Senior Staff Specialist, Royal North Shore Hospital

\section{Anthony Kull}

Registrar in Cardiology, Royal North Shore Hospital

The presence of a link between periodontal and cardiovascular disease is supported by epidemiological publications and studies of biological mechanism. Although still unproven, a causal relationship is attractive for several reasons. First, it fits with current thinking about atherosclerosis as an inflammatory disorder and the contribution from infective processes. Second, it suggests that current cardiovascular risk assessment could be improved by dental examination. Third, and of great potential clinical importance, prevention and treatment of periodontal disease could reduce cardiovascular disease (CVD).

Recent prospective cohort and case-control analyses by Beck and Loesche and their colleagues support this relationship. ${ }^{1,2}$ Beck analysed 1,147 men from the US Normative Aging and the Dental Longitudinal studies. ${ }^{1}$ Mean alveolar bone loss scores were significantly associated with total coronary heart disease (odds ratio 1.5), fatal coronary heart disease (1.9) and stroke (2.8). In a case-control study of 320 veterans who were either seen at a dental outpatient clinic $(n=206)$ or in a long-term nursing home $(n=114)$, Loesche found a statistically significant association between coronary heart disease and several oral health parameters, such as the number of missing teeth, plaque benzoyl-DL-argininenaphthylamide (BANA) test scores and salivary levels of Streptococcus sanguis. ${ }^{2}$

In epidemiological studies such as these, questions arise regarding potential confounders. Besides traditional risk factors such as age, cigarette smoking and diabetes mellitus that are common to both dental and CVD, both conditions are modified by factors such as level of education, income, stress and social isolation. Thus, the associations may primarily reflect the fact that people who are more health conscious are at lower risk of coronary heart disease and have better dental health. The decision to extract teeth, in addition to the condition of the teeth, is also influenced by financial considerations and access to health care, as well as attitudes about the value of oral health. Since these behaviours and attitudes are hard to measure, controlling for them is difficult and surrogates for healthy behaviour (for example, level of physical activity) should be considered. Beck and colleagues included level of education in their analytic models, along with age, body mass index, smoking status, blood pressure, family history of heart disease, cholesterol and alcohol consumption. Significant associations remained in the study by Beck as well as others that provided adjustment models, although questions remain as to how complete such adjustments are.

Diet is another possible explanation for the relationship between tooth loss and CVD. While a poor diet rich in sugar may lead to both periodontal disease and CVD, tooth 